\title{
Psychological impact of polygamous marriage on women and children: a systematic review and meta-analysis
}

\author{
Ismail Shaiful Bahari ${ }^{1}$, Mohd Noor Norhayati ${ }^{1 *}$, Nik Hussain Nik Hazlina², \\ Che Abd Aziz Mohamad Shahirul Aiman² and Nik Ahmad Nik Muhammad Arif ${ }^{2}$
}

\begin{abstract}
Background: Over the last two decades, there has been significant growth in public, political, and academic awareness of polygamy. Polygamous families have distinct household problems, usually stemming from jealousy between co-wives over the husband's affections and resources. This study aimed to ascertain the psychological impact of polygamous marriage on women and children worldwide.
\end{abstract}

Methods: A systematic search was performed in MEDLINE (PubMed), Scopus, CINAHL (EBSCOhost), Google Scholar, and ProQuest using search terms such as "marriage" and "polygamy." Studies published from the inception of the respective databases until April 2021 were retrieved to assess their eligibility for inclusion in this study. The Joanna Briggs Institute Critical Appraisal Checklist was used for data extraction and the quality assessment of the included studies. The generic inverse variance and odds ratios with 95\% confidence intervals (Cl) were calculated using RevMan software.

Results: There were 24 studies fulfilling the eligibility criteria, and 23 studies had a low risk of bias. The pooled meta-analysis showed women in polygamous marriages had a 2.25 (95\% Cl: 1.20,4.20) higher chance of experiencing depression than in monogamous marriages. Children with polygamous parents had a significantly higher Global Severity Index with a mean difference of $0.21(95 \% \mathrm{Cl}: 0.10,0.33)$ than those with monogamous parents.

Conclusions: The psychological impact of polygamous marriage on women and children was found to be relatively higher than monogamous marriage. Awareness of the proper practices for polygamy should be strengthened so that its adverse effects can be minimized. The agencies involved in polygamous practices should broaden and enhance their understanding of the correct practice of polygamy.

Keywords: Polygamy, Impact, Psychological, Social, Women, Children

\section{Background}

Polygamy may create a complex family system involving the husband's relationship and relations between subsequent wives and children [1]. Polygamous families have

\footnotetext{
*Correspondence: hayatikk@usm.my

${ }^{1}$ Department of Family Medicine, School of Medical Sciences, Universiti Sains Malaysia, Health Campus, 16150 Kubang Kerian, Kelantan, Malaysia Full list of author information is available at the end of the article
}

distinct household problems, usually stemming from jealousy between co-wives over the husband's affections and resources [2]. In addition to studies documenting polygamy's detrimental effects on wives' health, researchers have identified polygamy as a risk factor for adverse child health outcomes [3].

Polygamy is defined as "a marital relationship involving multiple spouses" [4]. There are three types of polygamy: polygyny refers to "one husband [who] is married to two 
or more wives," polyandry refers to "one wife married to two or more husbands," and polygynandry refers to "a group marriage scenario in which two or more wives are simultaneously married to two or more husbands" [4]. Only $2 \%$ of the global population practices polygamy. Polygamy is most often found in West and Central Africa, which the highest was in Burkina Faso (36\%) with widespread among people who practice folk religions (45\%), Muslims (40\%), and Christians (24\%) [5].

A recent systematic review had confirmed that children from polygamous marriages experienced physical and emotional abuse associated with parental neglect and abuse [6]. A qualitative study on female children and young adults found that polygamous marriage formed an emotional abuse to the daughters since they have witnessed the mother's severe pain of second marriage and ascribe the mother's pain to it [7]. These abuses may be associated with more mental health problems, social problems, and lower academic achievement in children from polygamous marriages compared to monogamous marriages [8].

In a qualitative study of American Muslims of various ethnic backgrounds, women in polygamous relationships have reported being abused by their husbands or other wives [3]. The prevalence of emotional distress (86.8\%), fearful feeling (17\%), low self-esteem (58.4\%), and loneliness $(64.1 \%)$ have also been found higher among women in polygamous relationships compared to monogamous marriages with the prevalence of $17.9,7.7,7.7$, and $12.8 \%$, respectively in Bedouin-Arabs of the Negev region in Israel [9] In polygamous marriages, where the mother is the first wife, the environment at home is stressful, parental investment is low, and resources are diluted; however, studies on polygamy and associated fertility issues have been mixed [10].

Polygamous women are genuinely at risk of experiencing psychological and emotional distress. For example, one study found that women in polygamous marriages are at a higher risk of low self-esteem and depression than women in monogamous relationships and enjoy less marital satisfaction and more problematic mother-child relationships [11]. There were significant differences between women in polygamous and monogamous marriages. There was a higher prevalence of somatization, depression, anxiety, hostility, paranoid ideation, psychoticism, general symptom severity, positive symptoms total, and psychiatric disorder, as well as lower ratings of life and marital satisfaction, family functioning, and selfesteem among polygamous wives [12]. A recent study also demonstrated similar findings but showed no significant difference in women's marital satisfaction between polygamous and monogamous marriages [13].
On the bright side, polygamy also demonstrated positive impacts. Childless wives are willing to have legal and valid polygamous marriages than the other wives to obtain offspring and descendants for the husband. Besides that, warmth and affection for polygamous families may provide positive role models for children's mental health and self-esteem [14].

Determining the impact of polygamous marriage on women and children worldwide can provide a better assessment than discrete primary studies. Identifying this impact can help give a clear understanding and serve as the basis for the development of appropriate strategies that address primary prevention to counter the potential negative impact affecting women and children. This systematic review and meta-analysis aimed to ascertain the psychological impact of polygamous marriage on women and children worldwide. We have included both women and children because the impact of polygamous marriage might affect both groups. This review summarizes the available evidence, effect estimates, and strength of the statistical associations between polygamous and monogamous marriages and the psychological impact on women, and children.

\section{Methods}

\section{Study design and search strategy}

A systematic review and meta-analysis were conducted to assess the impact of polygamous marriages on women and children. The Preferred Reporting Items for Systematic Reviews and Meta-Analyses (PRISMA) guidelines were followed [15]. This review was registered in the PROSPERO database (CRD42021226530). The review followed the process outlined in the protocol. A systematic search for relevant articles was performed in the MEDLINE (PubMed), Scopus, CINAHL (EBSCOhost), Google Scholar, and ProQuest databases. The search was undertaken using descriptors such as "marriage" (MeSH terms) OR "polygamy" (text word) AND "women" (MeSH terms) AND "children" (MeSH terms). The search terms were flexible and tailored to the various electronic databases. Studies published from the inception of the respective databases until April 2021 were retrieved to assess their eligibility for this study. The reference lists of the included citations were cross-checked to find additional potentially eligible studies.

\section{Eligibility criteria}

The inclusion criteria included studies that reported the psychological impact of polygamous marriage on women and children of all ages up to 18 years old. The Oxford dictionary defines psychological impact as involving the mental and emotional state of a person [16]. In this study, 
polygamy referred to "a marital relationship involving multiple wives" [4].

Studies with cross-sectional, case-control, and cohort designs published in English were included. Case series/ reports, conference papers and proceedings, articles available only in abstract form, editorial reviews, letters of communication, commentaries, systemic reviews, and qualitative studies were excluded.

\section{Study selection and screening}

All the records identified using our search strategy were exported to EndNote X8 software (Clarivate Analytics, Philadelphia, PA). Duplicate articles were removed. Two independent reviewers screened the titles and abstracts of the identified articles. The full texts of the eligible studies were obtained and read thoroughly to assess their suitability. A consensus discussion was held in a conflict between the two reviewers, and a third reviewer was consulted. The search method presented in the PRISMA flowchart (Fig. 1) shows the included and excluded studies, with reasons for the exclusions.

\section{Quality assessment and bias}

Critical appraisal was performed to assess the data quality using the Joanna Briggs Institute Critical Appraisal Checklist for cross-sectional, case-control, and cohort studies [6]. Two reviewers performed the bias assessments independently. The risk of bias was considered low when more than $70 \%$ of the answers were "yes," moderate when $50-69 \%$ of the answers were "yes," and high when up to $0-49 \%$ of the answers were "yes." Studies that showed a high or moderate risk of bias were excluded from the meta-analysis [17].

\section{Data extraction process}

Two reviewers independently extracted data into Microsoft Excel 365 (Microsoft, Redmond, Washington). The process included the first author, publication year, study location, study design and setting, study population, sample size, impact, polygamy definition, and data for calculation of effect estimates for psychological impact. In the event of missing data, the authors were contacted to obtain further information.

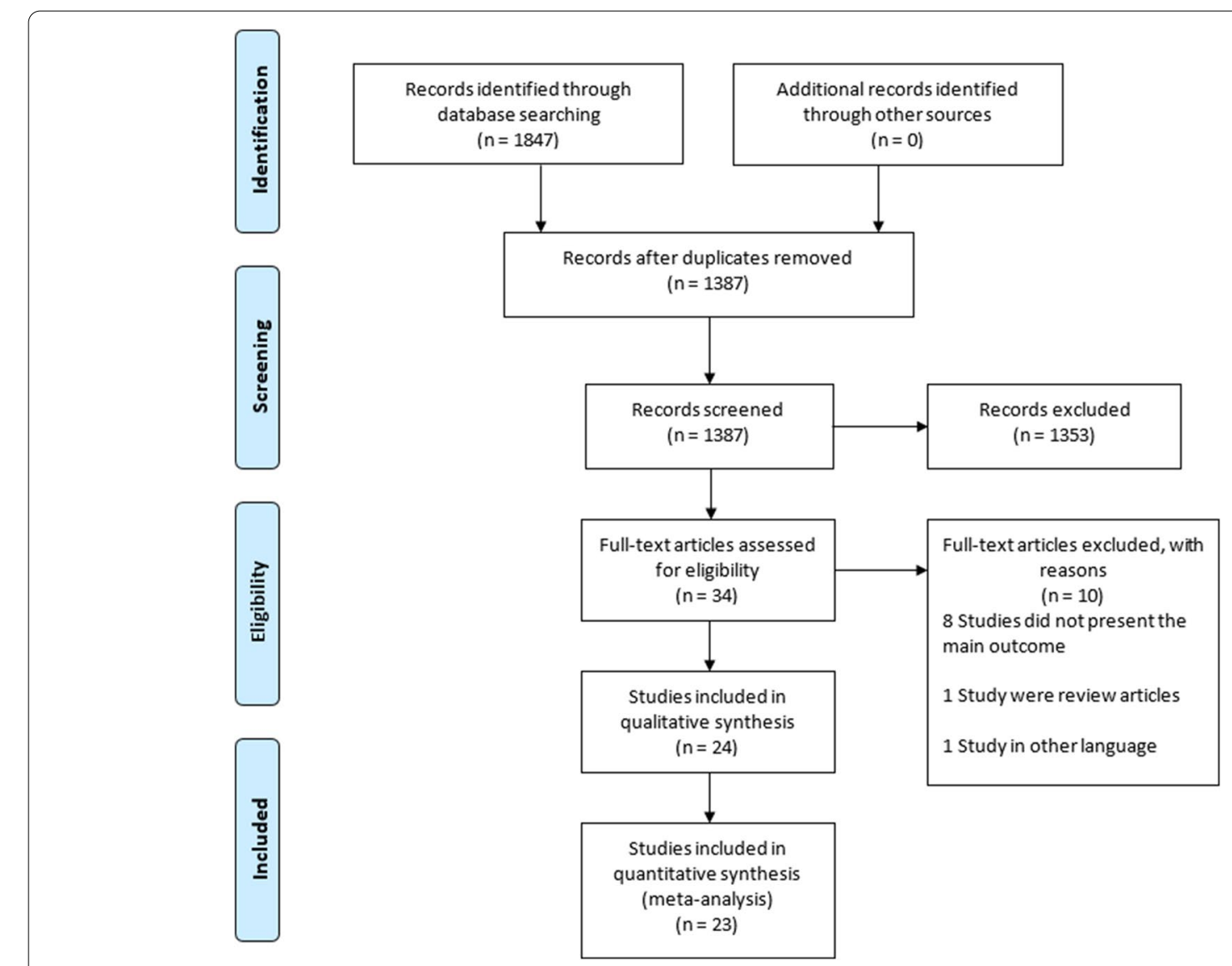

Fig. 1 Prisma flow chart impact of polygamous marriage on women and children 


\section{Results synthesis and statistical analysis}

The prevalence outcomes of the total sample over the total population were reported as percentages, and the cumulative estimates were reported as odds ratios (OR) and mean differences (MD) with $95 \%$ confidence intervals (CI). The analysis was performed using RevMan software version 5.4 (Nordic Cochrane Centre, Copenhagen, Denmark). We used a generic inverse variance with a random-effects model to pool the data. The $\mathrm{I}^{2}$ statistic was used to assess heterogeneity. As a guide, $\mathrm{I}^{2}$ was interpreted as follows: $0-40 \%$ might not be important, $30-60 \%$ may represent moderate heterogeneity, 50-90\% may represent substantial heterogeneity, and $75-100 \%$ indicated considerable heterogeneity [18]. The subgroup analyses were performed based on geographical regions if there was an adequate number of articles for each subgroup. Sensitivity analysis was conducted for studies with a wide range of confident intervals.

\section{Results}

\section{Characteristics of the included studies}

A total of 1847 articles were retrieved through the electronic database search using different search terms (Supplementary file 1), and 545 duplicated records were removed. The remaining 1387 articles were screened for eligibility. Among them, 1353 articles were excluded based on their titles and/or abstract evaluations. The full texts of 35 articles were searched. Subsequently, ten articles were excluded; where eight studies [19-26] did not present the main outcome, one study [14] was a review article, and one study [27] was in another language. Twenty-four studies underwent a quality assessment using Joanna Briggs Institute Critical Appraisal Checklist (Fig. 1, Supplementary file 2). Based on the quality assessment, 23 studies had a low risk of bias and one study had a moderate risk of bias [28]. All the low risk studies were cross-sectional and proceeded with quantitative assessment.

Among the 23 studies, 17 are about women [4, 9, 2943], while six other studies focus on the children [44-49]. Among the studies, 11 of them is from Israel [4, 9, 3032, 34, 44-46, 48, 49], three studies from Turkey [40, 41, 43], two studies from Iran [36, 38], a study from Uganda [29], a study from Nigeria [47], a study from Egypt [33], a study from UAE [37], a study from Syria [39], a study from Tanzania [42] and a study from Jordan [35]. The smallest sample size was 66 [38], and the largest was 2000 [35]. This study included 5963 women (Table 1) and 1567 children (Table 2).

\section{Prevalence of polygamy}

Seventeen studies were included for estimation of the prevalence of polygamy in the women population $[4,9$, 29-43]. A wide range was observed, ranging from $6.3 \%$ [39] to $66.7 \%$ [43]. The pooled prevalence of polygamy reported between 2001 and 2019, mainly in the

Table 1 Summary of research articles $(n=18)$ on the impact of polygamous marriages on women

\begin{tabular}{|c|c|c|c|c|c|c|}
\hline Authors & Study Area & Study design & Sample size (n) & $\begin{array}{l}\text { Polygamous } \\
\text { marriage (n) }\end{array}$ & $\begin{array}{l}\text { Monogamous } \\
\text { marriage (n) }\end{array}$ & $\begin{array}{l}\text { Quality } \\
\text { assessment } \\
(\%)\end{array}$ \\
\hline Abbo $2008[29]$ & Uganda & Cross-sectional & 209 & 37 & 90 & 100 \\
\hline Al- Sherbiny 2005 [33] & Egypt & Cross-sectional & 100 & 50 & 50 & 100 \\
\hline Daradkeh 2006 [35] & Jordan & Cross-sectional & 2000 & 544 & 947 & 100 \\
\hline Hamdan 2008 [37] & United Arab Emirates & Cross-sectional & 224 & 28 & 155 & 100 \\
\hline Kianpoor 2006 [38] & Iran & Cross-sectional & 66 & 31 & 26 & 75 \\
\hline Maziak 2002 [39] & Syria & Cross-sectional & 412 & 26 & 331 & 100 \\
\hline Ozkan 2006 [41] & Turkey & Cross-sectional & 138 & 88 & 50 & 100 \\
\hline Patil 2008 [42] & Tanzania & Cross-sectional & 408 & 96 & 312 & 87.5 \\
\hline Ozer 2013 [40] & Turkey & Cross-sectional & 172 & 99 & 73 & 87.5 \\
\hline Farahmand 2019 [36] & Iran & Cross-sectional & 398 & 248 & 150 & 100 \\
\hline Yilmaz 2018 [43] & Turkey & Cross-sectional & 108 & 72 & 36 & 100 \\
\hline Daoud 2014 [34] & Israel & Cross-sectional & 461 & 100 & 361 & 87.5 \\
\hline Al-Krenawi 2001 [4] & Israel & Cross-sectional & 92 & 53 & 39 & 100 \\
\hline Al-Krenawi 2006 [9] & Israel & Cross-sectional & 352 & 117 & 235 & 87.5 \\
\hline Al-Krenawi 2008 [32] & Israel & Cross-sectional & 315 & 156 & 159 & 100 \\
\hline Al-Krenawi 2011 [31] & Israel & Cross-sectional & 199 & 93 & 106 & 100 \\
\hline Al-Krenawi 2012 [30] & Israel & Cross-sectional & 309 & 187 & 122 & 100 \\
\hline Chaleby 1985 [28] & Kuwait & Cross-sectional & 125 & 31 & & 62.5 \\
\hline
\end{tabular}


Table 2 Summary of research articles $(n=6)$ on the impact of polygamous marriages on children

\begin{tabular}{|c|c|c|c|c|c|c|}
\hline Authors & Study Area & Study design & Sample size (n) & $\begin{array}{l}\text { Polygamous } \\
\text { marriage (n) }\end{array}$ & $\begin{array}{l}\text { Monogamous } \\
\text { marriage (n) }\end{array}$ & $\begin{array}{l}\text { Quality } \\
\text { assessment } \\
\text { (\%) }\end{array}$ \\
\hline Al-Krenawi, 2002 [44] & Israel & Cross-sectional & 101 & 19 & 82 & 87.5 \\
\hline Al-Krenawi 2000 [45] & Israel & Cross-sectional & 292 & 146 & 146 & 100 \\
\hline Al-Krenawi 2008 [46] & Israel & Cross-sectional & 352 & 178 & 174 & 100 \\
\hline Bamgbade 2014 [47] & Nigeria & Cross-sectional & 206 & 50 & 156 & 100 \\
\hline Hamdan, 2009 [49] & Israel & Cross-sectional & 406 & 208 & 198 & 87.5 \\
\hline Elbedour 2003 [48] & Israel & Cross-sectional & 210 & 114 & 84 & 75 \\
\hline
\end{tabular}

middle-east region, was $41.12 \%$ (95\% CI: $31.89,50.36$ ) (Fig. 2).

\section{Impact of polygamy on women compared to monogamy}

In this review, the psychological impact, including depression and anxiety, on women in polygamous marriages compared to monogamous marriages was evaluated. Only the pooled meta-analysis analysis for depression [34, 37, 40-43] showed a significant difference among women where it is 2.25 (95\% CI: 1.20, 4.20) higher chance of experiencing depression in polygamous marriages compared to monogamous marriages. However, for psychological distress (OR 1.57 [95\% CI: 0.60, 4.10]) $[29,39,42]$ and anxiety (OR 1.20 [95\% CI: 0.47,3.11]) [41-43] there were no significant difference between women in polygamous and monogamous marriages (Fig. 3). Panic disorder, too, did not show a significant difference (OR 4.05 [95\% CI: 0.71, 23.13]). Sensitivity analysis was conducted in the anxiety data due to the wide range of confident intervals in Yilmaz [43]. The estimated OR changed to 0.88 (95\% CI: $0.55,1.40)$ with $\mathrm{I}^{2}$ of $0 \%$.

Four studies [9, 30-32] evaluated a broad range of psychological impact using the Symptom Checklist90-Revised (SCL-90) instrument (Table 3). The scores for somatization (MD 0.50 [95\% CI: $0.28,0.72]$ ), obsessivecompulsive (MD 0.37 [95\% CI: 0.09, 0.64]), interpersonal sensitivity (MD 0.41 [95\% CI: 0.14, 0.67]), depression (MD 0.46 [95\% CI: 0.16, 0.77]), anxiety (MD 0.49 [95\% CI: $0.23,0.75]$ ), hostility (MD 0.49 [95\% CI: 0.25, 0.73]), phobia (MD 0.39 [95\% CI: 0.11, 0.67]), paranoia (MD 0.36 [95\% CI: 0.20, 0.51]), and psychoticism (MD 0.42 [95\% CI: 0.20, 0.64]) had significantly higher occurence in the women from polygamous marriages than monogamous

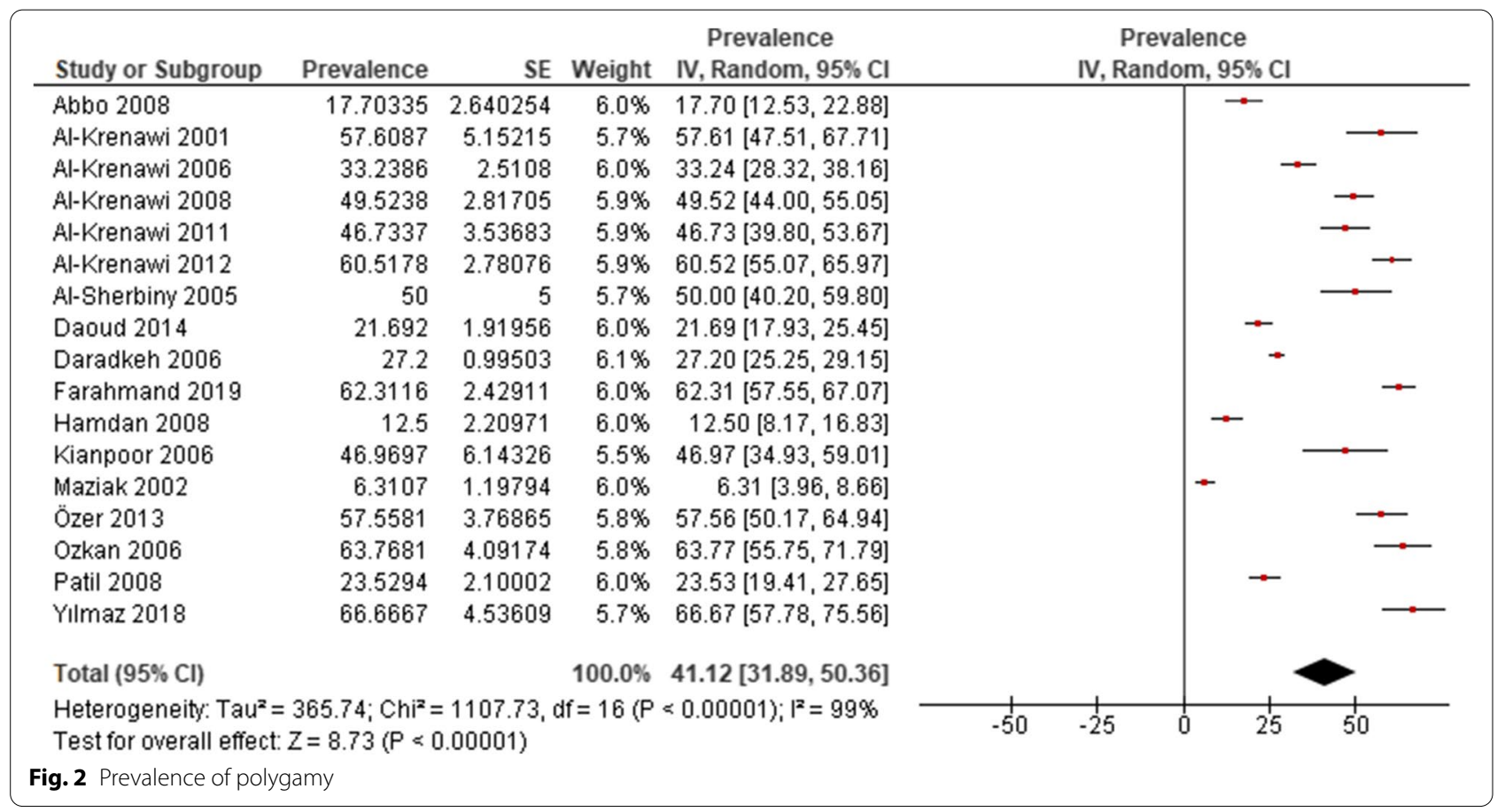




\section{(A) Psychological Distress}

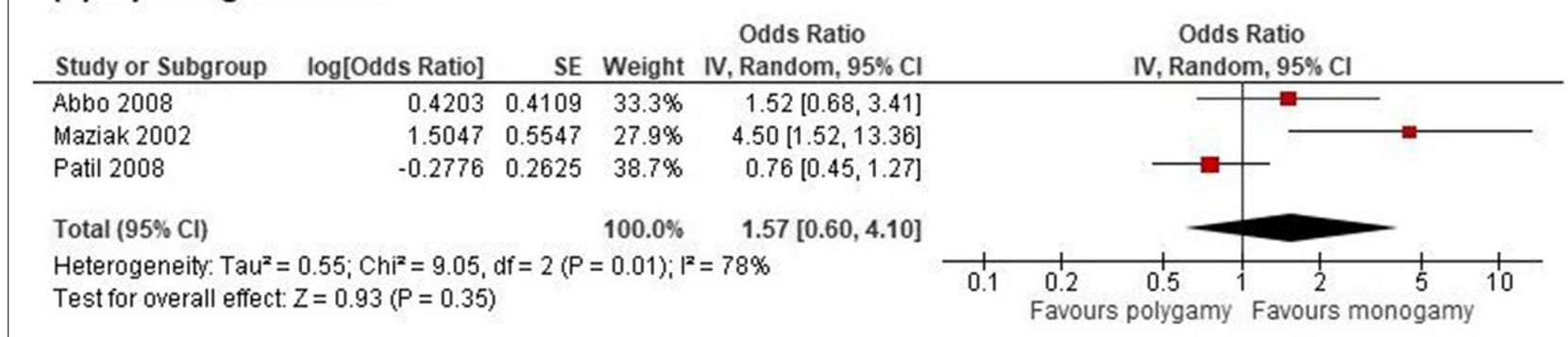

\section{(B) Depression}

\begin{tabular}{lrrrr} 
Study or Subgroup & log[Odds Ratio] & SE & Weight & $\begin{array}{c}\text { Odds Ratio } \\
\text { IV, Random, 95\% Cl }\end{array}$ \\
\hline Daoud 2014 & 0.6154 & 0.2324 & $19.6 \%$ & $1.85[1.17,2.92]$ \\
Hamdan 2008 & 0.6696 & 0.4219 & $15.8 \%$ & $1.95[0.85,4.47]$ \\
Ozer 2013 & 2.4383 & 0.4819 & $14.6 \%$ & $11.45[4.45,29.45]$ \\
Ozkan 2006 & 0.9567 & 0.4297 & $15.6 \%$ & $2.60[1.12,6.04]$ \\
Patil 2008 & -0.1795 & 0.2604 & $19.0 \%$ & $0.84[0.50,1.39]$ \\
Yilmaz 2018 & 0.7324 & 0.4413 & $15.4 \%$ & $2.08[0.88,4.94]$ \\
& & & & \\
Total (95\% Cl) & & $100.0 \%$ & $2.25[1.20,4.20]$ \\
Heterogeneity: Tau $^{2}=0.47 ;$ Chi $^{2}=24.32, d f=5(P=0.0002) ; I^{2}=79 \%$ \\
Test for overall effect: $Z=2.54(P=0.01)$ &
\end{tabular}

\section{(C) Anxiety}

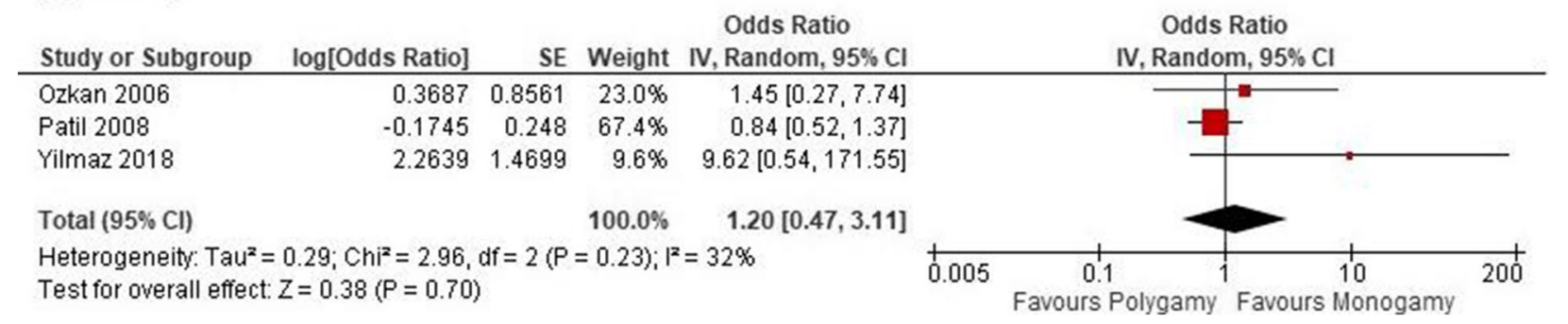

Fig. 3 Forest plots for $\mathbf{A}$ psychological distress, $\mathbf{B}$ depression, and $\mathbf{C}$ anxiety among women in polygamous versus monogamous marriages

Table 3 Mean differences in the Symptom Checklist-90-Revised scores among women in polygamous marriages versus those in monogamous marriages in four studies [9, 30-32]

\begin{tabular}{|c|c|c|c|c|}
\hline No. & $\begin{array}{l}\text { Symptoms assessed by the Symptom } \\
\text { Checklist-90-Revised }\end{array}$ & $\begin{array}{l}\text { Mean difference } \\
(95 \% \mathrm{Cl})\end{array}$ & Heterogeneity & $\begin{array}{l}P \text {-value of the } \\
\text { overall effect }\end{array}$ \\
\hline 1 & Somatization & $0.50(0.28,0.72)$ & $76 \%$ & $<0.001$ \\
\hline 2 & Obsessive-compulsive behavior & $0.37(0.09,0.64)$ & $87 \%$ & 0.009 \\
\hline 3 & Interpersonal sensitivity & $0.41(0.14,0.67)$ & $86 \%$ & 0.003 \\
\hline 4 & Depression & $0.46(0.16,0.77)$ & $92 \%$ & 0.003 \\
\hline 5 & Anxiety & $0.49(0.23,0.75)$ & $84 \%$ & $<0.001$ \\
\hline 6 & Hostility & $0.49(0.25,0.73)$ & $82 \%$ & $<0.001$ \\
\hline 7 & Phobia & $0.39(0.11,0.67)$ & $86 \%$ & 0.007 \\
\hline 8 & Paranoia & $0.36(0.20,0.51)$ & $54 \%$ & $<0.001$ \\
\hline 9 & Psychoticism & $0.42(0.20,0.64)$ & $81 \%$ & $<0.001$ \\
\hline 10 & Global Severity Index & $0.44(0.20,0.68)$ & $86 \%$ & $<0.001$ \\
\hline
\end{tabular}


marriages. Global Severity Index (GSI) for psychological dimensions is also higher in polygamous marriage compared to monogamous with a mean difference of 0.44 (95\% CI: 0.20, 0.68). Furthermore, four studies [9, 30-32] also reported on family function by using McMaster Family Assessment Device (FAD) among women where polygamous marriage had shown a mean difference of 0.34 (95\% CI: $0.20,0.49$ ) compared to monogamous marriages.

\section{Impact of polygamy on children compared to monogamy marriages}

There were two studies [44, 46] which reported the impact of polygamy in the children in terms of psychological impact using the SCL-90 instrument (Table 4). All scores for the psychological impact reported a slightly higher risk in children with parents practicing polygamy compared to monogamy where somatization (MD 0.20 [95\% CI: 0.07, 0.34]), obsessive-compulsive (MD 0.27 [95\% CI: 0.012, 0.42]), interpersonal sensitivity (MD 0.30 [95\% CI: 0.14, 0.46]), depression (MD 0.22 [95\% CI: 0.08, $0.37]$ ), anxiety (MD 0.07 [95\% CI: $-0.06,0.20]$ ) with $p>0.05$, hostility (MD 0.24 [95\% CI: 0.09, 0.39]), phobia (MD 0.33 [95\% CI: 0.18, 0.49]), paranoia (MD 0.16 [95\% CI: $0.01,0.31]$ ), and psychoticism (MD 0.28 [95\% CI: $0.12,0.43]$ ). The GSI for children with polygamous parents have higher mean difference which is 0.21 (95\% CI: $0.10,0.33$ ) compared to monogamous parents. In terms of social problems [44, 46], children with polygamous parents have higher risk of family dysfunction with $\mathrm{MD}$ 0.33 (95\% CI: - 0.11, 0.77) compared to monogamous marriage. For school achievement, two studies [45, 46] reported children with polygamous parents had lower scores compared to monogamous parents and a study [47] reported that children with polygamous parents had difficulties in understanding subjects such as Mathematics and English.

\section{Discussion}

The review was conducted to determine the psychological impact of polygamous marriage among women and children. The pooled prevalence of polygamous marriage in women from 17 studies was $41 \%$ (95\% CI: 32, 50). Among women, depression was found to be significantly different between polygamous and monogamous marriages. Women and children in polygamous marriages have higher scores in somatization, obsessive-compulsive, interpersonal sensitivity, anxiety, hostility, phobia, paranoia, psychoticism, and GSI compared to monogamous marriages.

Various research reported that first wives in polygamous marriages would have a higher risk of depression, anxiety, and negative attitude $[25,38,50,51]$. These researches reported similar findings as this current metaanalysis, where women in polygamous marriages have two times higher risk of developing depression compared to monogamous marriages. Al-Sherbiny [41] reported the "first wife syndrome," where the first wife reported difficulties faced psychological, physical, and social problems among women in a polygamous marriage. This syndrome goes through a course of reaction where the initial response from the first wife after being informed of her husband's remarriage is in the form of a nervous breakdown, emotional upset, or outburst of anger. Negative attitudes towards the husband and hostility towards the new wife always exist. After a lapse of time and gradual adaptation, these women reported that negative physical, psychological and social attitudes would decrease [33].

Al Krenawi [25] also reported that the transition from sole wife to senior wife is traumatic, leading to the senior wife having a loss of self-esteem. The Bedouin-Arabs of

Table 4 Mean differences in the Symptom Checklist-90-Revised scores among children with polygamous parents compared to monogamous parents in two studies $[44,46]$

\begin{tabular}{lllll}
\hline No. & $\begin{array}{l}\text { Symptoms assessed by the Symptom } \\
\text { Checklist-90-Revised }\end{array}$ & $\begin{array}{l}\text { Mean difference } \\
\mathbf{9 5 \%} \mathbf{C l})\end{array}$ & Heterogeneity & $\begin{array}{l}\text { P-value of the } \\
\text { overall effect }\end{array}$ \\
\hline 1 & Somatization & $0.20(0.07,0.34)$ & $0 \%$ & 0.003 \\
2 & Obsessive-compulsive behavior & $0.27(0.12,0.42)$ & $0 \%$ & $<0.001$ \\
3 & Interpersonal sensitivity & $0.30(0.14,0.46)$ & $0 \%$ & $<0.001$ \\
4 & Depression & $0.22(0.08,0.37)$ & $0 \%$ & 0.003 \\
5 & Anxiety & $0.07(-0.06,0.20)$ & $0 \%$ & 0.300 \\
6 & Hostility & $0.24(0.09,0.39)$ & $0 \%$ & $<.002$ \\
7 & Phobia & $0.33(0.18,0.49)$ & $0 \%$ & 0.001 \\
8 & Paranoia & $0.16(0.01,0.31)$ & $0 \%$ & $<0.001$ \\
9 & Psychoticism & $0.28(0.12,0.43)$ & $0 \%$ & $<0.001$ \\
10 & Global severity index & $0.21(0.10,0.33)$ & & \\
\hline
\end{tabular}


Negev showed that $58.4 \%$ of the polygamous wives had low self-esteem. This circumstance encouraged them to withdraw from their social networks, contributing to feeling lonely (64.1\%) among these polygamous wives.

Women in polygamous marriages scored significantly higher in all psychological dimensions in the SCL-90: somatization, interpersonal sensitivity, depression, anxiety, phobic, paranoia, psychoticism, and GSI, and these findings were similar to a review [13]. Al- Issa [52] indicated that somatization might be more prevalent in the non-western world than in the west. This may be due to the ethnicity of Arabs, where exhibiting somatization behaviour is one of the major ways to express emotional distress $[4,23,52]$. In this culture, the first wife is usually not consulted when her husband $\mathrm{s}$ to remarry, leading to fewer familial, social, and economic resources where it can be distressing [53]. This would lead to first wives in polygamous marriages having more anxiety, psychoticism, paranoia, and feeling of powerlessness than the second and third wives [22]. Apart from that, this metaanalysis also reports that family functioning scores have been worse in women with polygamous marriages than monogamous marriages. It may be due to the husband's attention being divided between two families; thus, economic resources became more diluted. One study reported that family functioning and financial status depend on one another, strongly associated with mental disorders [54]. A worsened family's economic situation could lead to poorer family functioning [32].

Children with polygamous parents experienced more psychological impact compared to monogamous parents; however, these findings were limited to only two studies. A review based on five papers concluded that children from polygamous families had higher levels of psychological impacts than those from monogamous families [8]. Elbedour [10] suggested that polygamy effects on children are more noticeable and disappear as they grow older. Children in polygamy marriages will have lower academic achievement [6, 24, 25]. Still, children's academic achievement may be less affected due to a better understanding of stressful events and more successful managing emotions [48]. Children from kindergarten through Grade 6 reported a lower level of education achievement based on the examination results. They had difficulty adjusting to their schools, thus indicating that these social problems were impacted by their parents' polygamous marriage that has affected their formal education system [45]. The children of these marriages will have a huge disadvantage in their education and increase school dropouts.

The SCL-90 instrument performed on the children in polygamous marriages showed higher psychological impact scores in all nine domains [44, 46]. However, there may be an additional cultural impact on some of the domains. Research revealed that Arab children exhibit higher levels of depression compared to the control samples in the United States [55]. It also implied interpersonal sensitivity, where its risk increased in conjunction with the presence of depression [56]. Despite having parents with polygamous or monogamous marriages, family functioning plays a much more prominent role in children's self-esteem, peer relation, and mental health $[44,46]$. Findings indicated the impact of polygamy itself, but a well-functioning family will not impair children's social adjustment and mental health [57]. Economic status also plays a significant role in family functioning and children's mental health $[46,58]$. Unfortunately, the children perceived that their parents' polygamous marriages had made their families' economic and family functioning worse $[44,46]$. This plays a major role in dealing with children's emotional and financial pressure.

This meta-analysis has a few limitations. Most studies have a very different range of tests and scales that hinder making a reasonable conclusion. The randomeffects model assumes the presence of heterogeneity in which each study has its study-specific effect. However, subgroup analysis to explore the differences to understand the observed effect was not possible due to limited studies. This study is limited by only including studies published in the English language. Most studies were conducted in the Middle East, specifically Arab societies, limiting the results and comparisons. We could not deduce whether the impact is solely due to polygamous marriages or the culture of societies. All the included studies were of cross-sectional design. Due to its nature, temporal causation cannot be established.

\section{Conclusions}

The psychological impact of polygamous marriage on women and children was relatively higher than monogamous marriage. This study also concluded that polygamous marriage plays a major role in the development of children not only mentally but also socially. Family functioning also has a major role in determining the outcome of polygamous impact on the population. Awareness of the proper practices for polygamy should be strengthened so that its adverse effects can be minimized. The agencies involved in polygamous practices should broaden and enhance their understanding of the correct practice of polygamy. It is also necessary for healthcare professionals to have a better evaluation for women and children in this family practice to provide them with a better quality of life. Polygamy should be recognized as a particular risk factor for developing social problems in children; thus, with proper education to the families, more attention to the children's emotional and social needs is required to 
avoid this situation. Future studies on polygamous marriage should emphasize more on children with broader sampling across various cultures. These studies should also use standardized measuring tools to ensure a better conclusion.

\section{Supplementary Information}

The online version contains supplementary material available at https://doi. org/10.1186/s12884-021-04301-7.

\section{Additional file 1.}

Additional file 2 .

\section{Acknowledgements}

The authors also would like to acknowledge Mr. Amran Mamat and Ms. Nuru Azurah Mohd Roni, librarians from Hamdan Tahir Library, for their assistance with database searches.

\section{Authors' contributions}

Conceptualization, ISB, MNN, and NHNH; methodology, ISB, MNN and NHNH; validation MNN and $\mathrm{NHNH}$; formal analysis, MNN, CAAMSA and NANMA: investigation, CAAMSA, and NANMA; resources, MNN and NHNH; data curation, NHNH, CAAMSA and NANMA; writing of original draft preparation, CAAMSA, and NANMA; writing of review and editing, ISB, MNN, NHNH, CAAMSA, and NANMA; visualization, ISB, MNN, NHNH; supervision, ISB and $\mathrm{NHNH}$; project administration, $\mathrm{NHNH}$; All authors have read and agreed to the published version of the manuscript.

\section{Availability of data and materials}

All data are available within the manuscript.

\section{Declaration}

Ethics approval and consent to participate

Not applicable.

\section{Consent for publication}

Not applicable.

\section{Competing interests}

The authors declare that they have no competing interests.

\section{Author details}

${ }^{1}$ Department of Family Medicine, School of Medical Sciences, Universiti Sains Malaysia, Health Campus, 16150 Kubang Kerian, Kelantan, Malaysia. 'Women's Health Development Unit, School of Medical Sciences, Universiti Sains Malaysia, Health Campus, Kubang Kerian, Kelantan, Malaysia.

Received: 24 June 2021 Accepted: 23 November 2021 Published online: 13 December 2021

\section{References}

1. Sinai M, Peleg O. Marital interactions and experiences of women living in polygamy: an exploratory study. Int J Psychol. 2021;56(3):361-77.

2. Adams BN, Mburugu E. Kikuyu bridewealth and polygyny today. J Comp Fam Stud. 1994;25(2):159-66.

3. Hassouneh-Phillips D. Polygamy and wife abuse: a qualitative study of Muslim women in America. Health Care Women Int. 2001;22(8):735-48.

4. Al-Krenawi A. Women from polygamous and monogamous marriages in an out-patient psychiatric clinic. Transcult Psychiatry. 2001;38(2):187-99.

5. Kramer S. 2020. 'Polygamy is rare around the world and mostly confined to a few regions', Pew Research Center. https://www.pewresearch.org/ fact-tank/2020/12/07/polygamy-is-rare-around-the-world-and-mostlyconfined-to-a-few-regions/.
6. Rose Chen Siew K, Siti Aishah H: Systematic review: agreement and impacts on wives and children well-being of polygamous family structure. J Crit Rev 2020, 7(13):596.

7. Alhuzail NA. Being a girl in a polygamous family implications and challenges. Child Adolesc Soc Work J. 2020;37(1):97-107.

8. Al-Sharfi M, Pfeffer K, Miller KA. The effects of polygamy on children and adolescents: a systematic review. J Child Fam Stud. 2016;22(3):272-86.

9. Al-Krenawi A, Graham JR. A comparison of family functioning, life and marital satisfaction, and mental health of women in polygamous and monogamous marriages. Int J Soc Psychiatry. 2006;52(1):5-17.

10. Elbedour S, Bart WM, Hektner JM. Scholastic achievement and family marital structure: Bedouin-Arab adolescents from monogamous and polygamous families in Israel. J Soc Psychol. 2000;140(4):503-14.

11. Brooks T. The problem with polygamy. Philos Top. 2009:109-22.

12. Shepard LD. The impact of polygamy on women's mental health: a systematic review. Epidemiol Psychiatr Sci. 2013;22(1):47-62.

13. Rahmanian P, Munawar K, Mukhtar F, Choudhry FR. Prevalence of mental health problems in women in polygamous versus monogamous marriages: a systematic review and meta-analysis. Arch Women's Ment Health. 2021;24(3):339-51.

14. Elbedour S, Onwuegbuzie AJ, Caridine C, Abu-Saad H. The effect of polygamous marital structure on behavioral, emotional, and academic adjustment in children: a comprehensive review of the literature. Clin Child Fam Psychol Rev. 2002;5(4):255-71.

15. Page MJ, McKenzie JE, Bossuyt PM, Boutron I, Hoffmann TC, Mulrow CD, et al. The PRISMA 2020 statement: an updated guideline for reporting systematic reviews. BMJ. 2021;372:n71.

16. Dictionary OE: Oxford English Dictionary. In: Simpson, Ja \&Weiner, Esc. 1989

17. Geta TG, Woldeamanuel GG, Dassa TT. Prevalence and associated factors of premenstrual syndrome among women of the reproductive age group in Ethiopia: systematic review and meta-analysis. PLoS One. 2020;15(11):e0241702.

18. Higgins JPT, Chandler J, Cumpston M, Li T, Page MJ, Welch VA: Cochrane handbook for systematic reviews of interventions version 6.1 (updated September 2020): Cochrane; 2020.

19. Al-Krenawi A, Graham JR, Ben-Shimol-Jacobsen S. Attitudes toward and reasons for polygamy differentiated by gender and age among BedouinArabs of the Negev. IJMHS. 2006;35(1):46-61.

20. Eapen V, Al-Gazali L, Bin-Othman S, Abou-Saleh M. Mental health problems among schoolchildren in United Arab Emirates: prevalence and risk factors. JAAPEE. 1998:37(8):880-6.

21. Elbedour S, Hektner JM, Morad M, Abu-Bader SH. Parent-adolescent conflict and its resolution in monogamous and polygamous Bedouin Arab families in southern Israel. Sci World J. 2003;3:1249-64.

22. Al-Krenawi A. Mental health and polygamy: the Syrian case. World J Psychiatry. 2013;3(1):1-7.

23. Al-Krenawi A, Graham JR. Somatization among Bedouin-Arab women: differentiated by marital status. J Divorce Remarriage. 2004;42(1-2):131-43.

24. Cherian VI. Academic achievement of children from monogamous and polygynous families. J Soc Psychol. 1990;130(1):117-9.

25. Al-Krenawi A, Graham JR, Al-Krenawi S. Social work practice with polygamous families. Child Adolesc Soc Work J. 1997;14(6):445-58.

26. Grych JH, Fincham FD. Marital conflict and children's adjustment: a cognitive-contextual framework. Psychol Bull. 1990;108(2):267.

27. Agustinawati M. Dinamika penerimaan diri pada istri pertama dalam pernikahan poligami: Universitas Islam Negeri Maulana Malik Ibrahim; 2020.

28. Chaleby K. Women of polygamous marriages in an inpatient psychiatric service in Kuwait. J Nerv Ment Dis. 1985;173(1):56-8.

29. Abbo C, Ekblad S, Waako P, Okello E, Muhwezi W, Musisi S. Psychological distress and associated factors among the attendees of traditional healing practices in Jinja and Iganga districts, eastern Uganda: a crosssectional study. IJMHS. 2008;2(1):1-9.

30. Al-Krenawi A. A study of psychological symptoms, family function, marital and life satisfactions of polygamous and monogamous women: the Palestinian case. Int J Soc Psychiatry. 2012;58(1):79-86.

31. Al-Krenawi A, Graham JR, Al Gharaibeh F. A comparison study of psychological, family function marital and life satisfactions of polygamous and monogamous women in Jordan. Community Ment Health J. 2011:47(5):594-602. 
32. Al-Krenawi A, Slonim-Nevo V. The psychosocial profile of Bedouin Arab women living in polygamous and monogamous marriages. Fam Soc. 2008;89(1):139-49.

33. Al-Sherbiny LAM. The case of first wife in polygamy: Description of an Arab culture-specific condition. ArabPsyNet 2005, No.8.

34. Daoud N, Shoham-Vardi I, Urquia ML, O'Campo P. Polygamy and poor mental health among Arab Bedouin women: do socioeconomic position and social support matter? Ethn Health. 2014;19(4):385-405.

35. Daradkeh T, Alawan A, Ma'aitah A, Otoom S. Psychiatric morbidity and its sociodemographic correlates among women in Irbid. EMHJ: Jordan; 2006.

36. Farahmand $M$, Rezvani Z. The association between father's power, performance, and mental stress of first wife in monogamous and polygamous families: a comparative study in Iran. Iran J Psychiatry Behav Sci 2019, $13(2)$.

37. Hamdan A, Hawamdeh S, Hussein A. The prevalence and correlates of depressive symptoms among Arab women in a primary health care setting. IJPM. 2008;38(4):453-67.

38. Kianpoor M, Bakhshani NM, Daemi N. Co-occurrence of panic disorder and being a wife in polygamous family. J Med Sci. 2006;6(5):870-3.

39. Maziak W, Asfar T, Mzayek F, Fouad FM, Kilzieh N. Socio-demographic correlates of psychiatric morbidity among low-income women in Aleppo, Syria. Soc Sci Med. 2002;54(9):1419-27.

40. Özer A, Orhan FÖ, Ekerbiçer HÇ. Sociodemographic variables and depression in Turkish women from polygamous versus monogamous families. Health Care Women Int. 2013;34(11):1024-34.

41. Ozkan M, Altindag A, Oto R, Sentunali E. Mental health aspects of Turkish women from polygamous versus monogamous families. Int J Soc Psychiatry. 2006:52(3):214-20.

42. Patil C, Hadley C. Symptoms of anxiety and depression and mother's marital status: an exploratory analysis of polygyny and psychosocial stress. Am J Hum Biol. 2008;20(4):475-7.

43. Yilmaz E, Tamam $L$. The relationship between polygamy and psychiatric disorders in Turkish women. Int J Soc Psychiatry. 2018;64(8):821-7.

44. Al-Krenawi A, Graham JR, Slonim-Nevo V. Mental health aspects of ArabIsraeli adolescents from polygamous versus monogamous families. J Soc Psychol. 2002;142(4):446-60.

45. Al-Krenawi A, Lightman ES. Learning achievement, social adjustment, and family conflict among Bedouin-Arab children from polygamous and monogamous families. J Soc Psychol. 2000;140(3):345-55.

46. Al-Krenawi A, Slonim-Nevo V. Psychosocial and familial functioning of children from polygynous and monogamous families. J Soc Psychol. 2008;148(6):745-64.

47. Bamgbade EO, Saloviita T. School performance of children from monogamous and polygamous families in Nigeria. J Black Stud. 2014;45(7):620-34.

48. Elbedour S, Bart WM, Hektner J. Intelligence and family marital structure: the case of adolescents from monogamous and polygamous families among Bedouin Arabs in Israel. J Soc Psychol. 2003;143(1):95-110.

49. Hamdan S, Auerbach J, Apter A. Polygamy and mental health of adolescents. Eur Child Adolesc Psychiatry. 2009;18(12):755-60.

50. Aghanwa HS, Dare FO, Ogunniyi SO. Sociodemographic factors in mental disorders associated with infertility in Nigeria. J Psychosom Res. 1999;46(2):117-23.

51. Al-Issa I. Culture and mental illness in Algeria. Int J Soc Psychiatry. 1990;36(3):230-40.

52. Al-Issa I. Culture and mental illness in an international perspective: International Universities Press, Inc; 1995.

53. Al-Krenawi A, Graham JR. The story of Bedouin-Arab women in a polygamous marriage. In: Women's Stud Int Forum: 1999: Elsevier; 1999: 497-509.

54. Viinamäki H, Kontula O, Niskanen L, Koskela K. The association between economic and social factors and mental health in Finland. Acta Psychiatr Scand. 1995;92(3):208-13

55. Lester D, Abdel-Khalek A. Suicidality and personality in American and Kuwaiti students. Int J Soc Psychiatry. 1998;44(4):280-3.

56. Boyce P, Parker G, Barnett B, Cooney M, Smith F. Personality as a vulnerability factor to depression. Br J Psychiatry. 1991;159(1):106-14.

57. Hoyle RH. Statistical strategies for small sample research: sage; 1999.

58. Beiser M, Hou F, Hyman I, Tousignant M. Poverty, family process, and the mental health of immigrant children in Canada. Am J Public Health. 2002:92(2):220-7.

\section{Publisher's Note}

Springer Nature remains neutral with regard to jurisdictional claims in published maps and institutional affiliations.
Ready to submit your research? Choose BMC and benefit from:

- fast, convenient online submission

- thorough peer review by experienced researchers in your field

- rapid publication on acceptance

- support for research data, including large and complex data types

- gold Open Access which fosters wider collaboration and increased citations

- maximum visibility for your research: over 100M website views per year

At BMC, research is always in progress.

Learn more biomedcentral.com/submissions 